\title{
População em situação de rua e COVID-19
}

\author{
Bruno Eduardo Freitas Honorato 1 \\ Ana Carolina S. Oliveira ${ }^{2}$ \\ 1 Universidade Federal de Alfenas / Instituto de Ciências Sociais Aplicadas, Varginha / MG - Brasil \\ 2 Instituto Federal de Ciência e Tecnologia do Sul de Minas Gerais, Inconfidentes / MG - Brasil
}

O objetivo deste trabalho é propor sugestões práticas para a atuação no atendimento à população em situação de rua diante da pandemia da COVID-19 no Brasil. Com base numa pesquisa qualitativa contendo entrevistas com profissionais da área, análise de informações levantadas num portal de notícias de amplitude nacional e levantamento de artigos, relatórios de pesquisa e documentos informativos de outros países, sugerimos algumas estratégias que podem contribuir para a atuação da gestão municipal, sobretudo no âmbito da assistência social e nos equipamentos de assistência às pessoas em situação de rua no Brasil. Nossos resultados são parciais, embora incluam um panorama comparativo que permite inclusão de estratégias, ao que parece, ainda não implementadas no país. Destacamos também algumas questões que precisam ser repensadas pelos governos federal, estadual e municipal diante da urgência da implementação de políticas durante a pandemia da COVID-19.

Palavras-chave: COVID-19; população em situação de rua; assistência social.

\section{Población en situación de calle y COVID-19}

El objetivo de este trabajo es proponer sugerencias prácticas para actuar en la asistencia a la población sin hogar que frente a la pandemia de COVID-19 en Brasil. Con base en una investigación cualitativa que contiene entrevistas con profesionales del área; análisis de información extraída de un portal nacional de noticias; y recopilación de artículos, informes de investigación y documentos de información de otros países; sugerimos algunas estrategias que pueden contribuir al desempeño de la gestión municipal, especialmente en el ámbito de la asistencia social y en el equipamiento de asistencia para las personas que viven en las calles en Brasil. Nuestros resultados son parciales, aunque incluyan un panorama comparativo que permite la inclusión de estrategias que, al parecer, aún no se han implementado en Brasil. También destacamos algunos problemas que los gobiernos federales, estatales y municipales deben repensar en vista de la urgencia de implementar políticas durante la pandemia de COVID-19. Palabras clave: COVID-19; población sin hogar; asistencia social.

\section{Homeless population and COVID-19}

The objective of this work is to propose practical strategies to support the homeless population during the COVID-19 pandemic in Brazil. It adopts qualitative research, based on interviews with professionals in the field, analysis of information collected on a national news online portal, and analysis of articles, research reports and information documents from other countries. The findings allow suggesting strategies for local governments, particularly regarding social assistance and equipment to support homeless people in Brazil. The results, although partial, include a comparative panorama that allows strategies that are unprecedented in Brazil. We also highlight some issues that need to be considered by the federal, state, and local governments in view of the urgency posed by the COVID-19 pandemic.

Keywords: COVID-19; homeless population; social assistance. 


\section{INTRODUÇÃO}

Em tempos difíceis em que temos de ficar isolados em casa, vem à tona a preocupação com aqueles que não têm onde morar. Construir políticas sociais para pessoas em situação de rua sempre foi desafiador no Brasil, assunto sobre o qual já nos debruçamos em outras oportunidades e por diferentes olhares (Honorato, 2014; Honorato \& Saraiva, 2016; Honorato \& Saraiva, 2017; Honorato, Saraiva, \& Silva, 2017). No entanto, o momento atual urge, e as mudanças provocadas no cenário mundial pelo novo coronavírus (COVID-19) exigem respostas rápidas e cada vez mais eficazes para a estabilização da situação mundial, em especial para populações que têm alta possibilidade de contaminação.

Identificado em dezembro de 2019 na cidade de Wuhan, na China, o novo coronavírus, designado SARS-CoV-2, pode infectar pessoas de qualquer idade, sendo as mais velhas e com doenças crônicas as que se encontram em maior risco (World Health Organization, 2020). As complicações graves e o número de mortes ao redor do mundo atualizam diariamente a preocupação mundial com o avanço geográfico do vírus e com o aumento do número de vítimas.

A condição das pessoas em situação de rua merece consideração especial, uma vez que essa população é naturalmente suscetível à infeção e pode correr maior risco de exposição graças às condições em que se encontra (Aguiar, Meireles, Rebelo, \& Barros, 2020). A situação de rua leva a uma grande vulnerabilidade social, com dificuldades reconhecidas de acesso ao sistema de saúde brasileiro (Hino, Santos, \& Rosa, 2018) e aos apoios sociais (Aguiar et al., 2020). Se considerarmos a suscetibilidade altíssima a infecções sintomáticas, hospitalização e fatalidade entre essa população, não apenas em razão da idade avançada, mas também do declínio físico acelerado e de problemas mentais que frequentemente resultam da exposição a riscos e elementos agressivos (Culhane, Treglia, Steif, Kuhn, \& Byrne, 2020), o coronavírus entre a população em situação de rua (PSR) aponta para uma tendência preocupante, com importantes implicações na saúde pública e nos recursos de assistência à saúde, uma vez que mesmo os casos mais leves de coronavírus entre essas pessoas exigem consideração de locais de isolamento e manejo (Baggett, Lewis, \& Gaeta, 2020).

Pessoas em situação de rua com menos de 65 anos têm mortalidade por todas as causas 5 a 10 vezes maior que a da população em geral (Baggett et al., 2013). A infecção por COVID-19 pode aumentar essa disparidade (Tsai \& Wilson, 2020). Para traçar um breve panorama sobre o que o índice de contaminação da PSR pode vir a ser, uma recente pesquisa de Baggett et al. (2020) apresentou, durante o período de observação de 15 dias, num total de 182 adultos, o diagnóstico positivo da COVID-19 numa frequência cumulativa estimada de 46,3 casos (intervalo de confiança de 95\% 39, 9-53, 3 casos) por mil pessoas, em 3 de abril de 2020.

Em comparação com a população domiciliada, nesse mesmo período, Massachusetts apresentou a frequência cumulativa de 1,9 caso por mil pessoas, e, em 2 de abril de 2020, Boston registrou uma frequência cumulativa de 1,8 caso por mil residentes. A realidade da PSR em nível internacional é, no mínimo, alarmante (Lima et al., 2020).

Passados mais de 10 anos desde a publicação da Política Nacional para Pessoas em Situação de Rua (Decreto $n^{\circ} 7.053,2009$ ), a condição de precariedade social dessa população no Brasil tem se agravado, especialmente a partir de 2017, após a crise econômica e política que o país viveu e com o crescente desemprego, que tem forçado famílias a migrar de cidade em busca de trabalho, além de outros fatores que já os atingiam, como dependência química, conflitos familiares, entre outros. O desafio no Brasil continua sendo o momento da implementação das políticas públicas para essa 
população (Hino et al., 2018). O número fornecido de pessoas em situação de rua pelo último Censo Pop, realizado entre 2007 e 2008, foi de 31.922 adultos (Pesquisa Nacional, 2008).

Hoje, no país, são mais de 119.636 famílias em situação de rua cadastradas no Cadastro Único para Programas Sociais do Governo Federal (Cortizo, 2019). A precarização da situação de rua e a alta possibilidade de contaminação pela COVID-19 nos convida a agir com urgência e traçar possíveis respostas ao desafio de lidar com a PSR durante a pandemia. Nosso objetivo neste trabalho é propor sugestões práticas o atendimento a essa população diante da pandemia da COVID-19 no Brasil.

Este trabalho foi estruturado em três partes: apresentação dos aspectos metodológicos da pesquisa, análise e discussão dos dados levantados e, por fim, considerações finais, com orientações e sugestões práticas para a atuação governamental junto à PSR durante a pandemia da COVID-19.

\section{ASPECTOS METODOLÓGICOS}

A metodologia se constitui na sistematização de práticas que buscam resolver as questões da pesquisa (Mattos, 2006). Para o problema que nosso objetivo empenha, traçamos as seguintes estratégias: (a) entrevistar profissionais que trabalham nos equipamentos de atendimento às pessoas em situação de rua, como gestores de Centro Pop, gestores de instituições de acolhimento e cuidadores sociais; (b) fazer um levantamento de notícias publicadas na imprensa em nível nacional relacionando a PSR à COVID-19; (c) levantar notas, relatórios de pesquisa e artigos já publicados sobre as estratégias de enfrentamento da pandemia em relação a essas pessoas em outros países. Tendo o tempo de pesquisa como principal limitador e levando em consideração a dinamicidade do tema, optamos por estratégias que julgamos mais efetivas, buscando equilibrar possibilidade de acesso e confiabilidade dos dados.

\subsection{Coleta dos Dados}

Para a estratégia (a) entrevistamos 2 profissionais que atuam na linha de frente da área, sendo 1 coordenadora de Centro Pop ${ }^{1}$ do interior de Minas Gerais e 1 cuidadora social do Distrito Federal. Entrevistamos também 2 atores de Organizações não Governamentais (ONGs), em Uberlândia e Uberaba, no interior de Minas Gerais, que lidam diretamente com a PSR em instituições de acolhimento e recuperação, ao passo que julgamos a contribuição desses atores relevante também para a construção de um contexto mais amplo de análise. Os profissionais foram escolhidos, em primeiro lugar, pelo contato com os pesquisadores em pesquisas anteriores e, depois, por indicação de atores ligados ao atendimento à PSR. Com o prazo e o acesso limitado da pesquisa durante a pandemia, o acesso aos atores foi feito por meio virtual.

Para a estratégia (b) valemo-nos de matérias publicadas no portal de notícias Globo.com, o mais acessado no Brasil hoje em dia (https://www.alexa.com/topsites/countries/BR, recuperado em 16 de abril de 2020). Foram consideradas somente matérias por escrito relacionadas à realidade brasileira. Optamos por não considerar vídeos e podcasts. Usamos combinações entre as seguintes palavras-chave na busca: "coronavírus", "moradores de rua", "pessoas em situação de rua”, "prefeitura",

\footnotetext{
${ }^{1}$ Como a coordenação do Centro Pop, em geral, é exercida por apenas uma pessoa, que poderia ser identificada caso informássemos a cidade em que atua, optamos por não expor o nome do município, a fim de evitar o comprometimento do anonimato. Vale ressaltar, porém, que não se trata das outras duas citadas: Uberlândia e Uberaba.
} 
"estado", "governo federal", “ONGs", "COVID-19" e "ações". O período de aplicação da busca foi entre 12 de dezembro de 2019, data de início do surto na China, e 7 de abril 2020. Entretanto, as notícias pesquisadas têm maior ocorrência entre 24 e 27 de março. Foram analisadas um total de $47^{2}$ notícias que apresentaram no título pelo menos duas das palavras-chave acima mencionadas combinadas no período estipulado para a pesquisa.

Para a estratégia (c) foram levantados artigos, relatórios de pesquisa e notas editoriais publicados em periódicos de circulação internacional, visando a uma base comparativa para as ações que propomos nas outras estratégias de pesquisa.

\subsection{Análise dos Dados}

Nosso intuito com as entrevistas na estratégia (a) foi criar uma base de interpretação dos dados coletados nas outras etapas que nos aproximasse do campo da prática por meio do contato com profissionais que lidam diretamente com as pessoas em situação de rua nos equipamentos governamentais. Por isso, não nos detemos a uma análise pormenorizada de elementos linguísticos, mas a um exame do significado semântico-pragmático da conversação, como sugere Mattos (2006), e uma exposição sintetizada dessas falas em meio a interpretação dos dados. Além disso, buscamos identificar elementos da prática da gestão desses equipamentos que, pela proximidade com o contexto da ação, poderiam destacar aspectos de inventividade e insurgência humanas no processo de implementação de medidas governamentais.

Os dados levantados pelas estratégias (b) e (c) foram analisados por meio de análise de conteúdo (Bardin, 2006), considerando as seguintes fases: pré-análise, com leitura flutuante de todas as manchetes disponíveis no site até o fim da coleta; escolha das notícias e definição do corpus de análise; leitura geral do material coletado; codificação dos dados para a formulação de categorias de análise utilizando o referencial teórico e as informações das entrevistas; recorte do material em unidades de registro (parágrafos e manchetes das notícias); estabelecimento de categorias seguindo os princípios de exclusão mútua (entre categorias), homogeneidade (dentro das categorias), pertinência da mensagem (não distorção) e objetividade (compreensão e clareza da categoria); agrupamento das unidades de registro em categorias comuns (categorias iniciais); categorização progressiva dos tipos de estratégia e ação (categorias finais); inferências e interpretação baseadas no referencial, nas entrevistas e nos dados comparativos. Ficaram evidentes as repetições de medidas que vêm sendo tomadas no âmbito municipal e estadual, motivo pelo qual consideramos cessar a coleta de dados levando em conta um critério de saturação.

$\mathrm{Na}$ estratégia (c), fizemos uma ampla busca de artigos que relacionassem COVID-19 e PSR nas plataformas Spell, Scielo e Google Scholar. Consideramos, para essa estratégia, rastrear a confiabilidade das fontes de artigos e relatórios publicados pelo index COVID-19 da plataforma Publons (https:// publons.com/publon/covid-19/?sort_by=date\&title=homeless, recuperado em 17 de abril de 2020), embora a urgência das publicações e o prazo restrito de pesquisa tenham nos levado a encontrar referência apenas dos artigos de Tsai e Wilson, (2020); Kirby, (2020) e Wood, Davies, e Khan (2020).

\footnotetext{
${ }^{2}$ Como o portal Globo.com não fornece o número de matérias publicadas no período estipulado da pesquisa, não mensuramos a quantidade exata de reportagens pré-analisadas. Entretanto, estimamos um valor acima de 150 textos como total realizado entre as diversas palavras-chave.
} 
Considerando a análise comparativa com os outros dados que analisamos no Brasil, julgamos plausível incluir também análise de Aguiar et al. (2020) das estratégias governamentais em Portugal e o documento do Centers for Disease Control and Prevention, publicado em 2 de abril de 2020, com orientações para prestadores de serviços sobre as pessoas em situação de rua durante a pandemia da COVID-19.

\section{DISCUSSÃO DOS DADOS}

No levantamento das notícias que relacionam a PSR à COVID-19, categorizamos e analisamos ações de 15 estados, em mais de 30 municípios, em todas as regiões do país (Sudeste, Nordeste, Centro-oeste, Norte e Sul). Apresentamos essas ações e estratégias no Quadro 1. Identificamos, como categorias finais, cinco tipos de estratégias básicas que municípios e estados têm tomado: 1) de prevenção, 2) de intervenção por parte dos municípios, 3) de conscientização, 4) de infraestrutura e 5) voltadas aos profissionais que lidam com a PSR nos municípios. Também identificamos inciativas dos órgãos de controle estaduais e federais, como Defensoria Pública de Estado e da União, que categorizamos como ações do Judiciário para diferenciá-las das estratégias municipais.

As ações foram agrupadas por semelhança em âmbito municipal e estadual, embora em algumas notícias sejam ressaltadas ações de organizações do terceiro setor, como ONGs que atuam com a PSR. Da mesma forma, diferenciamos as ações que estão sendo executadas daquelas que foram noticiadas como propostas ou planos da gestão municipal ou estadual. A maior parte das notícias informa sobre ações executadas, isto é, em fase de implementação, o que acreditamos ser um cenário otimista.

\subsection{Brasil}

Identificamos como medida mais frequente que vem sendo tomada por municípios e estados a adaptação dos pontos de acolhimento já existentes ou criados temporariamente, seguida de perto pela instalação de abrigos temporários destinados ao acolhimento da PSR. Destacam-se, portanto, as estratégias de infraestrutura como as mais frequentes. No que tange as estratégias de prevenção e conscientização, destacaram-se como ações mais frequentes no corpus analisado a angariação e a distribuição de itens de higienização e alimentação, bem como a orientação sobre a existência da doença e formas de prevenção, respectivamente. Para o desenvolvimento de técnicas e ações mencionadas, é evidente a relevância da articulação entre diferentes setores dentro do governo - captação de espaços culturais, religiosos, esportivos e educacionais para serem utilizados como estrutura de acolhimento - e entre governo e iniciativas voluntárias como ONGs e instituições - recebimento, preparação e distribuição de itens de limpeza e alimentação.

É interessante destacar ainda a diversidade de medidas de adaptação nos equipamentos de atendimento à PSR, desde as mais simples, como intensificação da higiene do ambiente e dos acolhidos, redução da capacidade de pessoas e afastamento dos beliches, até as mais complexas, como triagem de pessoas que apresentarem sintomas ou que compõem o grupo de risco, além de instalação de ambulatórios. Todos esses protocolos seguem as recomendações da Organização Mundial de Saúde (OMS) e do Ministério da Saúde. 


\section{QUADRO 1 ESTRATÉGIAS E AÇÕES PARA 0 ENFRENTAMENTO DA COVID-19 JUNTO À PSR NO BRASIL}

\begin{tabular}{|c|c|c|c|}
\hline Estratégias & Ações & Âmbito & Região \\
\hline \multirow{6}{*}{ Prevenção } & $\begin{array}{l}\text { Angariar e distribuir produtos de limpeza e itens de } \\
\text { alimentação em locais de aglomeração de PSR. }\end{array}$ & $\begin{array}{l}\text { Municipal, estadual } \\
\text { e voluntariado }\end{array}$ & $\begin{array}{l}\text { Sudeste, Norte, Sul } \\
\text { e Nordeste }\end{array}$ \\
\hline & $\begin{array}{l}\text { Ofertar refeições gratuitamente nos restaurantes populares } \\
\text { estaduais. }\end{array}$ & Estadual & Nordeste \\
\hline & Ofertar máscaras cirúrgicas para pessoas em situação de rua. & Municipal & Sudeste \\
\hline & Realizar censo para verificar a extensão da PSR*. & Municipal & Sudeste \\
\hline & $\begin{array}{l}\text { Identificar e mapear a PSR com suspeita da COVID-19, bem } \\
\text { como possíveis novos suspeitos. }\end{array}$ & Municipal & Sudeste \\
\hline & Desenvolver plano de contingência voltado para a PSR. & Municipal & Sudeste \\
\hline \multirow[t]{2}{*}{ Intervenção } & $\begin{array}{l}\text { Encaminhar a PSR com suspeita da COVID-19 para unidades } \\
\text { de saúde ou acionar o Samu, bem como monitorar tais } \\
\text { encaminhamentos. }\end{array}$ & Municipal & $\begin{array}{l}\text { Sudeste, Centro- } \\
\text { oeste e Norte }\end{array}$ \\
\hline & $\begin{array}{l}\text { Transportar a PSR em veículo próprio da equipe, atendidas às } \\
\text { recomendações para evitar contágio. }\end{array}$ & Municipal & Sudeste \\
\hline \multirow[b]{2}{*}{ Conscientização } & $\begin{array}{l}\text { Criar espaços de informação sobre a COVID-19 voltados para } \\
\text { a PSR com profissionais de saúde. }\end{array}$ & Municipal & Sudeste \\
\hline & $\begin{array}{l}\text { Orientar sobre a doença, sobre higiene e prevenção e sobre } \\
\text { pontos de acolhimento no município. }\end{array}$ & $\begin{array}{l}\text { Municipal, } \\
\text { voluntariado e } \\
\text { estadual }\end{array}$ & $\begin{array}{l}\text { Sudeste, Sul, } \\
\text { Nordeste e Centro- } \\
\text { oeste }\end{array}$ \\
\hline \multirow{6}{*}{ Infraestrutura } & Instalar pias em áreas de grande movimentação de pessoas. & Municipal & Sudeste \\
\hline & Disponibilizar recursos para aluguel de hotel popular. & Estadual & Norte \\
\hline & $\begin{array}{l}\text { Atender à PSR de modo normal ou ampliado (acesso à } \\
\text { moradia, à alimentação ou à higiene) por meio dos pontos de } \\
\text { acolhimento já existentes na cidade. }\end{array}$ & $\begin{array}{l}\text { Municipal, } \\
\text { voluntariado e } \\
\text { estadual }\end{array}$ & $\begin{array}{l}\text { Sudeste, Norte e } \\
\text { Nordeste }\end{array}$ \\
\hline & $\begin{array}{l}\text { Instalar abrigos temporários para aumentar o número de } \\
\text { vagas disponíveis para o acolhimento na cidade. }\end{array}$ & $\begin{array}{l}\text { Municipal e } \\
\text { estadual }\end{array}$ & $\begin{array}{l}\text { Sudeste, Nordeste, } \\
\text { Norte e Sul }\end{array}$ \\
\hline & $\begin{array}{l}\text { Abrir ou adaptar espaços específicos voltados exclusivamente } \\
\text { para a PSR incluída no grupo de risco, com suspeita da } \\
\text { COVID-19 ou sintomas gripais. }\end{array}$ & Municipal & Sudeste e Sul \\
\hline & $\begin{array}{l}\text { Adaptar pontos de acolhimentos e restaurantes populares de } \\
\text { acordo com as recomendações da OMS e do Ministério da Saúde. }\end{array}$ & $\begin{array}{l}\text { Municipal e } \\
\text { estadual }\end{array}$ & $\begin{array}{l}\text { Sudeste, Norte e } \\
\text { Nordeste }\end{array}$ \\
\hline \multirow[b]{2}{*}{ Trabalhadores } & Orientar voluntários que tenham contato direto com a PSR. & Voluntariado & Sudeste \\
\hline & $\begin{array}{l}\text { Disponibilizar materiais de higiene e EPIs para equipe técnica } \\
\text { em contato direto com os acolhidos. }\end{array}$ & Municipal & Sudeste \\
\hline
\end{tabular}

Notas: *Algumas das notícias trazem ações planejadas e não necessariamente implementadas, como no caso dessa, que se refere à possibilidade de um censo.

Fonte: Elaborado pelos autores. 
As ações identificadas no Quadro 1 estão sendo planejadas e/ou executadas em diferentes municípios e estados, mas nenhum deles, até o momento, implementou todas elas - a maioria pôs em prática não mais do que quatro das ações acima citadas.

Medidas de outros órgãos que visam acionar o Poder Executivo - como Defensoria Pública do Estado, da União, juízes da Fazenda Pública, Ministério Público do Estado - apareceram com frequência como recomendações para o reforço de proteção e assistência à PSR, com ou sem detalhamento de ações a serem tomadas, e para o estabelecimento de prazos de adoção de recomendações ou manifestações. Embora haja casos omissos, muitas das iniciativas seguem as orientações desses órgãos. Em alguns, esses documentos exigem um posicionamento de prefeituras e governos estaduais em relação à PSR.

Segundo os entrevistados, a maioria das iniciativas tomadas nos municípios não vem de ordens oficiais do governo municipal, e sim dos próprios profissionais, que, trabalhando na linha de frente com a PSR, pesquisam individualmente sobre medidas e cuidados necessários à prevenção da contaminação, movimentando a gestão municipal a fim de cumprir as demandas específicas dessa população, como a disponibilização de espaços extras para isolamento e acolhimento.

O nível de informação repassada pelos governos tanto federal - o que inexiste no caso da PSR em específico - quanto estadual e municipal foi bastante questionado pelos entrevistados. Nenhuma orientação foi dada pelo governo federal sobre cuidados e estratégias de ação para lidar com essa população, a despeito do evidente risco de contaminação e transmissão do vírus. Um dos entrevistados, de uma ONG em Uberlândia, afirmou receber orientações da gestão municipal, mas esse depoimento foi o único entre os que recebemos sobre o assunto.

Uma das entrevistadas relatou que o Ministério da Saúde chegou a disponibilizar um curso de prevenção não diretamente voltado para a PSR. Mas, com a demanda extraordinária de trabalho e o número de funcionário reduzido - tanto pela necessidade de rotatividade quanto por afastamentos de profissionais do grupo de risco -, seria impossível ter acessar a ele.

Em Brasília, a situação parece bem mais complexa do que no interior, segundo os relatos a que tivemos acesso por entrevista. A falta de cuidados básicos e a desarticulação entre os equipamentos de assistência do governo do Distrito Federal têm gerado desgaste nos trabalhadores do setor, que precisam tomar iniciativa sobre as decisões que deveriam ser informadas com antecedência. $\mathrm{O}$ tema acerca da saúde mental dos trabalhadores surgiu especialmente forte nas entrevistas com profissionais dos equipamentos governamentais, não sendo citado pelos entrevistados das ONGs. O desamparo dos governos com esses profissionais é preocupante tanto no nível básico de oferecimento de instruções gerais de atendimento e EPI quanto no nível emocional, já que esses especialistas se encontram em ambiente de risco, de falta de força de trabalho por conta dos afastamentos e sob pressão para atendimentos e acolhimentos forçados de pessoas que já haviam sido suspensas por conta de agressões ou embriaguez, entre outros motivos.

A desarticulação entre os equipamentos é também um tema de atenção. Mesmo no interior, o nível de desarticulação entre Centros de Atenção Psicossocial a Álcool e Outras Drogas (Caps AD) e Centros Pop afeta diretamente os atendimentos. Também as condições de atendimento e o nível de acesso que a PSR tem ao Sistema Único de Saúde (SUS) preocupa os entrevistados, uma vez que o atendimento a essas pessoas muitas vezes envolve embriaguez e outras drogas que não são consideradas responsabilidade do SUS, mas dos órgãos ligados à PSR. Mesmo com sintomas, nem sempre a PSR é atendida por conta da embriaguez ou do uso de outras drogas. 
Outro tema que surgiu nas entrevistas e merece destaque é a impossibilidade de muitas pessoas de receber o benefício social de 600 reais oferecido pelo governo federal, haja vista que, ainda que conste no Cadastro Único (CadÚnico), a PSR precisa apresentar os documentos pessoais para receber o benefício, algo muitas vezes impossível, já que essa população nem sempre tem documentos. Houve uma desconsideração do governo federal sobre essa especificidade no momento de planejamento do benefício.

\subsection{Breve Panorama Internacional}

Após um rápido levantamento sobre o enfrentamento a COVID-19 voltado à PSR no cenário mundial, sintetizamos a seguir as principais estratégias identificadas em quatro países: Inglaterra, Austrália, Portugal e Estados Unidos.

A Inglaterra vem elaborando um plano, já aprovado pelo governo, focado na proteção aos vulneráveis, na redução da transmissão do vírus e na mitigação dos surtos (Kirby, 2020). O plano destina 3,2 milhões de libras para o financiamento inicial de emergência a pessoas que precisarem se autoisolar e procura esvaziar os aparelhos de acolhimento da PSR à medida que os acolhidos forem sendo destinados às instalações próprias para o isolamento, como hotéis não utilizados (Kirby, 2020).

Portugal, por sua vez, assegura a continuidade dos serviços de atendimento, acolhimento e distribuição de alimentos à PSR, adotando medidas como preparação de espaços para quarentena, além de conscientização, orientação sobre a pandemia e instruções para os trabalhadores da área, visando controlar a propagação do vírus nos aparelhos de acolhimento (Aguiar et al., 2020).

A Austrália, de modo similar ao Brasil, tem desafios que vão desde a disponibilização de condições de higiene simples, como lavar as mãos, até a garantia de possibilidades de isolamento e acesso aos serviços de saúde e EPIs para essa população (Wood et al., 2020). Apesar dos obstáculos, até o momento, porém, o país parece não ter apresentado um plano voltado especificamente para a PSR (Kirby, 2020), assim como os Estados Unidos (Tsai \& Wilson, 2020).

Os autores sugerem testagem e treinamento para a identificação da COVID-19 e iniciativas voltados para a PSR, bem como espaços alternativos que permitam o isolamento e o tratamento dessa população.

\subsection{Sugestões Práticas para o Atendimento à PSR Durante a Pandemia da COVID-19}

Nesta seção, apresentamos um compêndio de sugestões práticas para gestores municipais e equipe técnica que lidam com a PSR no Brasil. Tal compêndio foi desenvolvido com base nos dados que analisamos e fortemente inspirados no documento publicado pelo Center for Disease Control and Prevention (CDC), especialmente voltado para o enfrentamento da COVID-19 junto à PSR, considerando as especificidades do caso brasileiro.

As propostas são agrupadas de acordo com três possíveis situações: antes, durante e após um surto da COVID-19 entre a PSR local e de acordo com as estratégias identificadas por meio da análise do corpus. Para o momento atual brasileiro, como ainda não identificamos nenhum surto da COVID-19 junto à PSR, criamos o Quadro 2, focado no planejamento de ações para evitar surtos. Os Quadros 3 e 4 - durante e após o surto, respectivamente - foram traduzidos e adaptados do documento supramencionado, com a inclusão de categorias específicas. 


\section{QUADRO 2 SUGESTÕES PARA 0 PLANEJAMENTO DE AÇÕES CONTRA 0 COVID-19 ANTES DE UM SURTO}

\section{Estratégias de prevenção}

- Forneça suprimentos de prevenção aos equipamentos de assistência à PSR, como sabão, álcool em gel, lenços, copos descartáveis, máscaras cirúrgicas ou artesanais.

- Estabeleça comunicação contínua entre o departamento de saúde pública local e os equipamentos de atendimento à PSR, a fim de facilitar o acesso a informações relevantes antes e durante um surto.

- Faça um plano de emergência para ajudar a reduzir o impacto do surto. Durante o processo de planejamento, possibilite que prestadores de serviços e pessoas em situação de rua possam discutir e colaborar.

- Os equipamentos devem construir alianças fortes antes de um surto, a fim de fornecer à organização o suporte e os recursos necessários para responder de maneira eficaz.

- Forneça uma lista dos principais contatos nos departamentos de saúde locais e estaduais para os equipamentos.

- Inclua planos de contingência para lidar com 0 afastamento de outros trabalhadores. Considere contratar profissionais temporários no caso de um surto.

- Promova a prática de ações preventivas cotidianas. Use mensagens e materiais de saúde desenvolvidos por fontes credíveis de saúde pública, como os departamentos de saúde pública locais e estaduais.

- Identifique PSR que podem estar em alto risco de complicações da COVID-19 - mais velhos ou que tenham problemas de saúde subjacentes - para garantir que suas necessidades sejam levadas em consideração.

- Planeje um maior uso de abrigo durante o surto.

- Aborde as principais estratégias de prevenção em seu plano de operações de emergência. Revise-o e atualize-o sempre que possivel.

\section{Estratégias de intervenção}

- Transporte a PSR em veículo próprio dos equipamentos, atendidas as recomendações para evitar contágio.

- Os equipamentos devem estar preparados para relatar casos de doenças respiratórias ao seu departamento de saúde local e para transportar pessoas com doenças graves a instalações médicas. Monitore tais encaminhamentos.

\section{Estratégias de conscientização}

- Comunique sobre a COVID-19 e ações preventivas diárias.

- Crie um plano de comunicação para distribuir informações oportunas e precisas durante um surto.

- Identifique e elimine possíveis barreiras linguísticas, culturais e de deficiência associadas à comunicação de informações da COVID-19 a trabalhadores, voluntários e atendidos.

- Ajude a combater o estigma e a discriminação em sua comunidade. Fale contra comportamentos negativos e se envolva com grupos estigmatizados.

- Compartilhe seus planos com funcionários, voluntários, principais parceiros e partes interessadas da comunidade, solicitando feedback sobre eles. 
Estratégias para infraestrutura

- Adapte pontos de acolhimentos e restaurantes populares de acordo com as recomendações da OMS e do Ministério da Saúde.

- Quando possível, instale pias para lavagem das mãos em áreas de grande movimentação de pessoas.

- Mantenha o atendimento à PSR de modo normal ou ampliado - acesso à moradia, à alimentação ou à higiene - por meio dos pontos de acolhimento já existentes na cidade.

- Identifique espaços que podem ser usados para acomodar PSR com sintomas respiratórios leves e separe-os dos outros.

- Disponibilize recursos para ações emergenciais dos equipamentos, como instalação de abrigos temporários.

- Considere abrir ou adaptar espaços específicos voltados exclusivamente para PSR com suspeita da COVID-19 ou sintomas gripais e, quando possível, para os que estão no grupo de risco.

Estratégias de suporte para os trabalhadores

- Forneça EPIs para prevenção da COVID-19 aos profissionais que trabalham em contato direto com a PSR.

- Desenvolva materiais de treinamento e educação sobre os planos para funcionários e voluntários.

- Forneça serviços que garantam a saúde mental dos trabalhadores. Isso é de extrema importância para a efetividade do atendimento.

Fonte: Elaborado pelos autores.

\section{QUADRO 3 SUGESTÕES PARA O PLANEJAMENTO DE AÇÕES CONTRA A COVID-19 DURANTE DE UM SURTO}

\section{Estratégias de prevenção}

- Se casos forem relatados em sua comunidade, coloque seu plano de emergência em ação, a fim de proteger atendidos, funcionários e convidados.

- Mantenha-se informado sobre a situação local. Obtenha informações atualizadas sobre a atividade local da COVID-19 junto a autoridades de saúde pública.

- Implemente ações preventivas diárias e forneça instruções aos funcionários sobre ações para evitar a propagação da doença. Reúna-se com os gestores dos equipamentos de modo a discutir planos para ajudar os atendidos a implementar medidas preventivas pessoais.

- Os funcionários e os voluntários do grupo de risco - mais velhos ou que tenham problemas de saúde subjacentes não devem ser designados como cuidadores de doentes hospedados no abrigo.

- Se os funcionários lidarem com os pertences dos atendidos, devem usar luvas descartáveis.Certifique-se de treinar qualquer equipe que use luvas.

- No check-in, forneça a qualquer atendido com sintomas respiratórios (tosse, febre) uma máscara cirúrgica.

- Monitore os atendidos que podem pertencer ao grupo de risco - mais velhos ou que tenham problemas de saúde subjacentes - e os contate regularmente.

- Considere reduzir ao estritamente necessário a limpeza em quartos e banheiros dedicados a doentes, a fim de evitar o contato desnecessário com eles.

- Se identificar qualquer atendido com sintomas graves, solicite atendimento médico imediato. 


\section{Estratégias de conscientização}

- Mantenha a PSR e os trabalhadores informados sobre as recomendações de saúde pública, para evitar a propagação da doença e as alterações nos serviços que possam estar relacionados ao surto.

- Coloque placas nas entradas e em locais estratégicos, fornecendo instruções sobre higiene das mãos, higiene respiratória e etiqueta da tosse.

\section{Estratégias para infraestrutura}

- Minimize o número de funcionários que interajam cara a cara com atendidos que apresentem sintomas respiratórios.

- Use barreiras físicas para proteger a equipe que interage com atendidos com status de infecção desconhecido.

- Limite o número de visitantes à instalação.

- Em áreas de dormir em geral - para aqueles que não apresentam sintomas respiratórios -, assegure-se de que as camas (ou os colchões) estejam a uma distância apropriada umas das outras e solicite que todos os atendidos durmam com a cabeça voltada para o lado oposto ao da cama anterior.

- Forneça acesso a fluidos, tecidos e sacolas plásticas para o descarte adequado dos tecidos usados.

- Certifique-se de que banheiros e pias sejam consistentemente abastecidos com sabão e materiais secantes para lavar as mãos. Forneça desinfetantes para as mãos que contenham pelo menos 70\% de álcool, se for uma opção em seu abrigo, em pontos-chave da instalação, incluindo mesas de registro, entradas/saídas e áreas de alimentação.

- Mantenha os atendidos com sintomas respiratórios leves em salas individuais, se possível, e evite áreas comuns.

- Se salas individuais para atendidos doentes não estiverem disponíveis, considere usar um local grande e bem ventilado.

- Nas áreas em que os atendidos com doenças respiratórias estão hospedados, mantenha as camas afastadas a pelo menos um metro e oitenta, use barreiras temporárias entre elas, como cortinas, e solicite que todos os atendidos durmam com a cabeça voltada para o lado oposto ao da cama anterior.

- Se possível, designe um banheiro separado para atendidos com sintomas da COVID-19.

- Certifique-se de que todas as áreas comuns dentro da instalação sigam boas práticas de limpeza ambiental.

\section{Estratégias de suporte para os trabalhadores}

- Incentive funcionários e voluntários doentes a ficar em casa (ou a ir para casa se desenvolverem sintomas enquanto estiverem na unidade), a fim de evitar transmitir a infecção a outras pessoas.

- Forneça serviços que garantam a saúde física e mental dos trabalhadores. Isso é de extrema importância para a efetividade do atendimento.

Fonte: Traduzido e adaptado de Interim Guidance for Homeless Service Providers (2020). 


\section{QUADRO 4 SUGESTÕES PARA O PLANEJAMENTO DE AÇÕES CONTRA A COVID-19 APÓS UM SURTO}

\section{Estratégias de acompanhamento}

- Lembre-se de que um surto da COVID-19 pode durar muito tempo e 0 impacto em suas instalações pode ser considerável.

- Reserve um tempo para conversar sobre suas experiências com gestores, funcionários e atendidos.

- Avalie a eficácia do plano de ação da sua organização.

- Discuta e anote as lições aprendidas. Suas ações de preparação para a COVID-19 foram eficazes na organização? Fale sobre os problemas encontrados no seu plano e as soluções eficazes.

- Participe de discussões da comunidade sobre planejamento de emergência. Informe os outros sobre quais ações de prontidão funcionaram. Mantenha linhas de comunicação com sua comunidade - por exemplo, mídias sociais e listas de e-mail.

- Continue praticando ações preventivas diárias.

- Mantenha e expanda seu planejamento de emergência. Procure maneiras de expandir as parcerias da comunidade. Identifique agências ou parceiros necessários para ajudá-lo a se preparar para um surto de doença infecciosa no futuro.

Fonte: Traduzido e adaptado de Interim Guidance for Homeless Service Providers (2020).

\section{CONCLUSÃO}

Nosso objetivo neste trabalho foi propor sugestões práticas para o enfrentamento da COVID-19 junto à PSR no Brasil. Para tanto, optamos por uma abordagem qualitativa contendo entrevistas com profissionais da área, análise de informações levantadas num portal de notícias de amplitude nacional e levantamento de artigos, relatórios de pesquisa e documentos informativos de outros países. Sintetizamos essas sugestões nos Quadros 2, 3 e 4 deste trabalho e esperamos, com isso, contribuir para a atuação da gestão municipal e dos equipamentos de assistência à PSR no país.

Para além das sugestões, levantamos algumas questões que precisam ser repensadas pelos governos federal, estadual e municipal, as quais, com base em nossos dados, reiteramos nestas considerações finais:

1. O governo federal brasileiro ainda não se pronunciou quanto à criação e à implementação de políticas emergenciais para o apoio aos órgãos de assistência social no enfrentamento a COVID-19 junto à PSR. Consideramos que tal postura do governo é, no mínimo, negligente com relação a esse grupo social em específico.

2. A maioria das iniciativas que têm sido tomadas nos municípios não vem de ordens oficiais do governo municipal, e sim dos próprios profissionais que trabalham em instituições de assistência ou nas ONGs que auxiliam a PSR. Acreditamos que uma iniciativa do governo federal e uma maior cobrança dos órgãos do Poder Judiciário possam fazer agir os gestores municipais que ainda não se posicionaram sobre a PSR em seus municípios. 
3. Com demanda extraordinária de trabalho, número de funcionários reduzidos por conta dos afastamentos do grupo de risco, desarticulação entre equipamentos e ONGs, falta de EPIs em muitos equipamentos, e obrigados a tomar iniciativas e decisões sobre as ações de enfrentamento a COVID-19 sem amparo oficial dos governos municipal, estadual e federal, os profissionais da assistência se encontram muitas vezes sobrecarregados, o que pode comprometer sua saúde física e mental, além de reduzir o quadro de trabalhadores disponíveis. Por isso, acreditamos firmemente que esses profissionais precisam de amparo imediato do governo em cada uma dessas deficiências citadas.

4. A impossibilidade de muitas pessoas em situação de rua de receber o benefício social de $\mathrm{R} \$ 600,00$ oferecido pelo governo federal evidencia uma falha na implementação dessa política que precisa ser revista a tempo de incluir a PSR para maior amparo diante da situação emergencial.

Por fim, entendemos como limitação deste trabalho a carência de mais entrevistas com profissionais da linha de frente numa maior variedade de municípios do país e num maior aporte de dados de outros países, o que se configura como uma possibilidade de ampliação desta pesquisa no futuro. Com base nos relatos que obtivemos, argumentamos, acima de tudo, sobre as faltas que precisam ser preenchidas na construção de políticas emergenciais, esperando que essas lacunas possam ser completadas em breve por uma revisão consistente.

Embora haja avanços no acesso e na produção do cuidado à PSR no SUS, a precarização no acesso e na violação do direito à saúde dessa população tem sido constantemente relatada por pesquisadores da área (Paula \& Laura, 2018). A PSR do Brasil precisa, sobretudo neste momento de pandemia, não só de planos, mas de efetividade na implementação de estratégias de enfretamento. 


\section{REFERÊNCIAS}

Aguiar, A., Meireles, P., Rebelo, R., \& Barros, H. (2020). Covid-19 e as pessoas em situação de semabrigo: ninguém pode ser deixado para trás. In $\mathrm{M}$. Tavares \& C. Silva (Orgs.), Da emergência de um novo vírus humano à disseminação global de uma nova doença: doença por coronavírus 2019 (Covid-19). (Capítulo IX, pp. 1-6). Porto, Portugal: Universitário de São João; Universidade do Porto. Recuperado de http://asset.youoncdn.com/ab296ab30c207ac6 41882479782c6c34/7c7b39d50c8250c4b32f991c32 45e5f7.pdf

Baggett, T. P., Hwang, S. W., O'Connell, J. J., Porneala, B. C., Stringfellow, E. J., Orav, E. J., ... Rigotti, N. A. (2013). Mortality among homeless adults in Boston: shifts in causes of death over a 15 -year period. JAMA internal medicine, 173(3), 189-195.

Baggett, T. P., Lewis, E., \& Gaeta, J. M. (2020). COVID-19 outbreak at a large homeless shelter in Boston: Implications for universal testing. medRxiv, Preprint. Recuperado de https://www.medrxiv.org/ content/early/2020/04/15/2020.04.12.20059618

Bardin, L. (2006). Análise de conteúdo. Lisboa, Portugal: Edições 70. (Obra original publicada em 1977).

Cortizo, R. M. (2019). População em situação de rua no Brasil: o que os dados revelam? Brasília, DF: Ministério de Cidadania.

Culhane, D., Treglia, D., Steif, K., Kuhn, R., \& Byrne, T. (2020). Estimated Emergency and Observational/ Quarantine Capacity Need for the US Homeless Population Related to Covid-19 Exposure by County; Projected Hospitalizations, Intensive Care Units and Mortality. Los Angeles, CA: UCLA Campuswide Homelessness Initiative. Recuperado de: https:// escholarship.org/uc/item/9g0992bm

Decreto $N^{\circ} 7.053$ de 23 de dezembro de 2009. (2009). Institui a Política Nacional para a População em Situação de Rua e seu Comitê Intersetorial de Acompanhamento e Monitoramento, e dá outras providências. Brasília, DF: Presidência da República.

Hino, P., Santos, J. O., \& Rosa, A. S. (2018). Pessoas que vivenciam situação de rua sob o olhar da saúde. Revista Brasileira de Enfermagem, 71(supl1), 732-740.

Honorato, B. E. F. (2014). Ordem e subversão nas cidades: Um estudo sobre a população em situação de rua de Belo Horizonte (Dissertação de Mestrado). Universidade Federal de Minas Gerais, Belo Horizonte.

Honorato, B. E. F., \& Saraiva, L. A. S. (2016). Cidade, População em Situação de Rua e Estudos Organizacionais. Desenvolvimento em Questão, 14(36), 158-186.

Honorato, B. E. F., \& Saraiva, L. A. S. (2017). Quando a Casa é a Marquise, o Albergue, a Rua: discursos e políticas sociais para pessoas em situação de rua em Belo Horizonte. Administração Pública e Gestão Social, 9(4), 244-309.

Honorato, B. E.; Saraiva, L. A. S., \& Silva, E. R. (2017). A construção social da ordem e da subversão nos discursos da (e sobre a) população em situação de rua de Belo Horizonte. Revista Organizações em Contexto, 13(26), 339-383.

Interim Guidance for Homeless Service Providers. (2020, 25 de março). Centers for Disease Control and Prevention. Departament of Health \& Human Services. Recuperado de https://www.cdc.gov/ coronavirus/2019-ncov/communication/guidancelist.html?Sort=Date\%3A\%3Adesc

Kirby, T. (2020, 26 de março). Efforts escalate to protect homeless people from COVID-19 in UK. The Lancet Respiratory Medicine. Recuperado de https://www. thelancet.com/journals/lanres/article/PIIS22132600(20)30160-0/fulltext\#articleInformation

Lima, N. N. R., Souza, R. I., Feitosa, P. W. G., Moreira, J. L. S., Silva, C. G. L., \& Neto, M. L. R. (2020). People experiencing homelessness: Their potential exposure to COVID-19. Psychiatry Research, 288, 112945.

Mattos, P. L. C. L. (2006). Análise de entrevistas não estruturadas: da formalização à pragmática da linguagem. In A. Silva, C. Godoi, \& R. Bandeirade-Mello (Orgs.), Pesquisa qualitativa em estudos organizacionais: paradigmas, estratégias e métodos (pp. 347-373). São Paulo, SP: Saraiva.

Paula, M. D. S., \& Laura, C. M. F. (2018, January). População em situação de rua: seus (des) encontros com a saúde, construção de visibilidades, protagonismo e possibilidades de garantia de direitos sociais. In Proceedings of 2018 Convención Internacional de Salud, Havana, Cuba. Recuperado de: http://www.convencionsalud2018.sld.cu/index. php/connvencionsalud/2018/paper/view/1805/818 
Pesquisa Nacional sobre a População em Situação de Rua. (2008). (Sumário Executivo). Brasília, DF: Ministério do Desenvolvimento Social e Combate à Fome.

Tsai, J., \& Wilson, M. (2020). Covid-19: a potential public health problem for homeless populations. The Lancet Public Health, 5(4), e186-e187.
Wood, L., Davies, A., \& Khan, Z. (2020). Covid-19 precautions-easier said than done when patients are homeless. The Medical Journal of Australia, Preprint.

World Health Organization. (2020, 05 de janeiro). Pneumonia of unknown cause - China. (Disease outbreak News). Recuperado de https://www. who.int/csr/don/05-january-2020-pneumonia-ofunkown-cause-china/en/

\section{Bruno Eduardo Freitas Honorato}

https://orcid.org/0000-0003-3670-0095

Doutor em Administração pela Universidade Federal de Minas Gerais (UFMG); Professor Adjunto do Instituto de Ciências Sociais Aplicadas na Universidade Federal de Alfenas (Unifal-MG). E-mail: brunoefh@gmail.com

\section{Ana Carolina S. Oliveira}

https://orcid.org/0000-0003-0081-3741

Mestre em Administração pela Universidade Federal de Minas Gerais (UFMG); Professora EBTT do Instituto Federal de Ciência e Tecnologia do Sul de Minas Gerais (IF Sul de Minas).

E-mail: carolina.oliveira@ifsuldeminas.edu.br 